\title{
Leptogenesis Bound on Spontaneous Symmetry Breaking of Global Lepton Number
}

\author{
Pei-Hong $\mathrm{Gu}^{1}$ * and Utpal Sarkar对 \\ ${ }^{1}$ The Abdus Salam International Centre for Theoretical Physics, Strada Costiera 11, 34014 Trieste, Italy \\ ${ }^{2}$ Physical Research Laboratory, Ahmedabad 380009, India
}

\begin{abstract}
We propose a new class of leptogenesis bounds on the spontaneous symmetry breaking of global lepton number. These models have a generic feature of inducing new lepton number violating interactions, due to the presence of the Majorons. We analyzed the singlet Majoron model with right-handed neutrinos and find that the lepton number should be broken above $10^{5} \mathrm{GeV}$ to realize a successful leptogenesis because the annihilations of the right-handed neutrinos into the massless Majorons and into the standard model Higgs should go out of equilibrium before the sphaleron process is over. We then argue that this type of leptogenesis constraint should exist in the singlettriplet Majoron models as well as in a class of R-parity violating supersymmetric Majoron models.
\end{abstract}

PACS numbers: 98.80.Cq, 14.60.Pq, 12.60.Fr

There have been many experiments establishing the tiny but nonzero neutrino masses although we are yet to find out if the neutrinos are Majorana or Dirac particles. If the neutrinos are Majorana particles, lepton number is necessarily broken. In the simplest extension of the standard model (SM) for the small neutrino masses, the lepton number is explicitly broken by the supplement of a Weinberg dimension-5 operator [1] in the Lagrangian. This operator can be generated in the renormalizable seesaw 2] scenario, where an explicit lepton number breaking Majorana mass term for the right-handed neutrinos 2] and(or) trilinear interaction for the triplet and doublet Higgs scalars [3] are(is) introduced to the SM.

The seesaw models also provide a solution to the puzzle of the matter-antimatter asymmetry in the universe through leptogenesis [4]: the CP-violating and outof-equilibrium decays of the right-handed neutrinos [4] and(or) triplet Higgs scalars [5, 6, 7] to the SM particles can generate a lepton asymmetry, which is partially converted to a baryon asymmetry through the sphaleron [8] action and hence accounts for the matter-antimatter asymmetry. For this purpose, the Yukawa interactions of the right-handed neutrinos while the gauge, Yukawa and trilinear interactions of the triplet Higgs scalars should satisfy the out-of-equilibrium condition before the sphaleron process becomes very weak at $T_{s p h} \sim 100 \mathrm{GeV}$.

If we further consider that the original seesaw scheme to be originating from a more fundamental theory, where the lepton number is spontaneously broken locally or globally, there will be some new particles coupling to the right-handed neutrinos and(or) triplet Higgs scalars. This implies additional out-of-equilibrium conditions for a successful leptogenesis, which, in turn, constrains the breaking scale of the lepton number. For example, in the left-right symmetric model, the right-handed gauge bosons should be heavier than $10^{6-7} \mathrm{GeV}$ to guarantee the departure from equilibrium of the right-handed neu-

*Electronic address: pgu@ictp.it

${ }^{\dagger}$ Electronic address: utpal@prl.res.in trinos [5].

In this note, we first study the constraint from leptogenesis on the singlet Majoron model of Chikashige, Mohapatra and Peccei [9]. In this model, a singlet scalar, driving the spontaneous breakdown of the global lepton number conservation, is introduced to the SM in addition to the three right-handed neutrinos. The right-handed neutrinos couple to the singlet scalar and hence obtain a Majorana mass term after the lepton number is broken. These couplings also result in some scattering processes for the right-handed neutrinos to the light species. Therefore, the breaking scale of the lepton number conservation should be under the authority of the successful leptogenesis. We then extend this analysis to the singlettriplet Majoron model and the R-parity violating supersymmetric Majoron model, where this new leptogenesis constraint is also applicable for certain range of the parameters.

We start with the singlet Majoron model. In this model, the kinetic and Yukawa terms of the lepton sector would be

$$
\begin{aligned}
\mathcal{L}_{K} \supset & \overline{\psi_{L}} i \gamma^{\mu} D_{\mu} \psi_{L}+\overline{\ell_{R}} i \gamma^{\mu} D_{\mu} \ell_{R}+\overline{N_{R}} i \gamma^{\mu} \partial_{\mu} N_{R} \\
& +\left(\partial_{\mu} \chi\right)^{\dagger}\left(\partial^{\mu} \chi\right) \\
\mathcal{L}_{Y} \supset & -y_{\ell} \overline{\psi_{L}} \tilde{\phi} \ell_{R}-y_{\nu} \overline{\psi_{L}} \phi N_{R}-\frac{1}{2} h \chi \overline{N_{R}^{c}} N_{R}+\text { H.c. }(2)
\end{aligned}
$$

Here $\psi_{L}\left(\mathbf{1}, \mathbf{2},-\frac{1}{2}\right)$ and $\ell_{R}(\mathbf{1}, \mathbf{1},-1)$, respectively, are the SM lepton doublets and singlets, $\phi\left(\mathbf{1}, \mathbf{2},-\frac{1}{2}\right)$ is the SM Higgs doublet, $N_{R}(\mathbf{1}, \mathbf{1}, 0)$ denotes the right-handed neutrinos, $\chi(\mathbf{1}, \mathbf{1}, 0)$ is the Higgs singlet, where the transformations are given under the SM gauge group $S U(3)_{c} \times$ $S U(2)_{L} \times U(1)_{Y}$. Conventionally, we assign the Higgs singlet $\chi$ a lepton number $L=-2$, as the right-handed neutrinos $N_{R}$ have $L=+1$. As a result, the Yukawa interaction (2) is lepton number conserving since $L=1$ for the SM leptons $\psi_{L_{\alpha}}$ and $\ell_{R}$ while $L=0$ for the SM Higgs $\phi$. The general scalar potential contains the quadratic and quartic terms as below,

$$
\begin{aligned}
V(\chi, \phi)= & -\mu_{1}^{2}\left(\chi^{\dagger} \chi\right)+\lambda_{1}\left(\chi^{\dagger} \chi\right)^{2}-\mu_{2}^{2}\left(\phi^{\dagger} \phi\right) \\
& +\lambda_{2}\left(\phi^{\dagger} \phi\right)^{2}+\lambda_{3}\left(\chi^{\dagger} \chi\right)\left(\phi^{\dagger} \phi\right),
\end{aligned}
$$


where $\lambda_{3}>-2\left(\lambda_{1} \lambda_{2}\right)^{\frac{1}{2}}$ so that the potential is bounded from below.

Once the Higgs singlet $\chi$ develops its vacuum expectation value $(\mathrm{VEV})$, we can write

$$
\chi=\frac{1}{\sqrt{2}}(f+\sigma) e^{i \frac{\eta}{f}},
$$

where $f=\left(\mu_{1}^{2} / \lambda_{1}\right)^{\frac{1}{2}}$ is the VEV, $\sigma$ is the physical Higgs boson with the mass

$$
M_{\sigma}=\left(2 \lambda_{1}\right)^{\frac{1}{2}} f
$$

while $\eta$ is the massless Majoron. By taking the phase rotations,

$$
\begin{aligned}
e^{i \frac{\eta}{2 f}} N_{R} & \rightarrow N_{R}, \\
e^{i \frac{\eta}{2 f}} \psi_{L} & \rightarrow \psi_{L}, \\
e^{i \frac{\eta}{2 f}} \ell_{R} & \rightarrow \ell_{R},
\end{aligned}
$$

we can perform the following interactions,

$$
\begin{aligned}
\mathcal{L} \supset & \frac{1}{2 f}\left(\overline{\psi_{L}} \gamma^{\mu} \psi_{L}+\overline{\ell_{R}} \gamma^{\mu} \ell_{R}+\overline{N_{R}} \gamma^{\mu} N_{R}\right) \partial_{\mu} \eta \\
& +\frac{1}{f} \sigma \partial^{\mu} \eta \partial_{\mu} \eta-\left[y_{\ell} \overline{\psi_{L}} \tilde{\phi} \ell_{R}+y_{\nu} \overline{\psi_{L}} \phi N_{R}\right. \\
& \left.+\frac{1}{2 \sqrt{2}} h(f+\sigma) \overline{N_{R}^{c}} N_{R}+\text { H.c. }\right]-\lambda_{3} f \sigma \phi^{\dagger} \phi .
\end{aligned}
$$

For convenience, we choose the basis in which the Yukawa couplings $h \rightarrow \hat{h}$ are real and diagonal by the proper phase rotation and then define the Majorana neutrinos,

$$
N_{i}=N_{R_{i}}+N_{R_{i}}^{c}
$$

with the masses,

$$
M_{N_{i}}=\frac{1}{\sqrt{2}} \hat{h}_{i} f .
$$

We then obtain

$$
\begin{aligned}
\mathcal{L} \supset & -\left(y_{\nu} \overline{\psi_{L}} \phi N+\text { H.c. }\right)-\frac{1}{2} M_{N} \bar{N} N \\
& +\frac{1}{4 f} \bar{N} \gamma^{\mu} \gamma_{5} N \partial_{\mu} \eta-\frac{1}{2 \sqrt{2}} \hat{h} \sigma \bar{N} N \\
& +\frac{1}{f} \sigma \partial^{\mu} \eta \partial_{\mu} \eta-\lambda_{3} f \sigma \phi^{\dagger} \phi .
\end{aligned}
$$

Here the first line definitely indicates the conventional leptogenesis scenario in the seesaw context, the second and third lines result in the pair annihilations of the Majorana neutrinos to the Majoron and the SM Higgs as shown in Figs. 1 and $2{ }^{1}$.

\footnotetext{
${ }^{1}$ Before the electroweak symmetry breaking, the couplings of the SM leptons $\psi_{L}$ and $\ell_{R}$ to the Majoron $\eta$ have no contributions to the annihilations of the Majorana neutrinos $N$.
}

After an explicit calculations, we find the annihilation cross sections to be

$$
\begin{aligned}
& \sigma_{N_{i} N_{i} \rightarrow \eta \eta}|\vec{v}| \\
= & \frac{1}{32 \pi} \hat{h}_{i}^{4} \frac{1}{s}\left\{\frac{s^{2}\left(s-4 M_{N_{i}}^{2}\right)-8 M_{N_{i}}^{2} s\left(s-M_{\sigma}^{2}\right)}{M_{N_{i}}^{2}\left[\left(s-M_{\sigma}^{2}\right)^{2}+M_{\sigma}^{2} \Gamma_{\sigma}^{2}\right]}-4\right\} \\
& -\frac{1}{16 \pi} \hat{h}_{i}^{4} \frac{1}{\sqrt{s\left(s-4 M_{N_{i}}^{2}\right)}}\left[1+\frac{2 s\left(s-M_{\sigma}^{2}\right)}{\left(s-M_{\sigma}^{2}\right)^{2}+M_{\sigma}^{2} \Gamma_{\sigma}^{2}}\right] \\
& \times \ln \frac{\sqrt{s}-\sqrt{s-4 M_{N_{i}}^{2}}}{\sqrt{s}+\sqrt{s-4 M_{N_{i}}^{2}}}, \\
& \sigma_{N_{i}} N_{i} \rightarrow \phi^{*} \phi|\vec{v}| \\
= & \frac{1}{2 \pi} \lambda_{3}^{2} \frac{M_{N_{i}}^{2}\left(s-4 M_{N_{i}}^{2}\right)}{s\left[\left(s-M_{\sigma}^{2}\right)^{2}+M_{\sigma}^{2} \Gamma_{\sigma}^{2}\right]},
\end{aligned}
$$

where

$$
|\vec{v}|=2\left(1-\frac{4 M_{N_{i}}^{2}}{s}\right)^{\frac{1}{2}}
$$

is the relative velocity with $s$ being the squared center of mass energy. The total cross section is then given by

$$
\sigma_{A}|\vec{v}|=\sigma_{N_{i} N_{i} \rightarrow \eta \eta}|\vec{v}|+\sigma_{N_{i} N_{i} \rightarrow \phi^{*} \phi}|\vec{v}|
$$

In order to determine the frozen temperature $T_{F}$, at which the annihilation of $N_{i}$ becomes slower than the expansion of the Universe, we should calculate the thermal average cross section,

$$
\left\langle\sigma_{A}|\vec{v}|\right\rangle=\frac{\int_{4 M_{N_{i}}^{2}}^{\infty} s \sqrt{s-4 M_{N_{i}}^{2}} K_{1}\left(\frac{\sqrt{s}}{T}\right) \sigma_{A}|\vec{v}| d s}{\int_{4 M_{N_{i}}^{2}}^{\infty} s \sqrt{s-4 M_{N_{i}}^{2}} K_{1}\left(\frac{\sqrt{s}}{T}\right) d s}
$$

according to the standard manner [10, 11], and then require the reaction rate,

$$
\Gamma=n_{N_{i}}^{\mathrm{eq}}\left\langle\sigma_{A}|\vec{v}|\right\rangle
$$

with the equilibrium number density

$$
n_{N_{i}}^{\mathrm{eq}}=\frac{1}{\pi^{2}} M_{N_{i}}^{2} T K_{2}\left(\frac{M_{N_{i}}}{T}\right),
$$

to be smaller the Hubble constant,

$$
H(T)=\left(\frac{8 \pi^{3} g_{*}}{90}\right)^{\frac{1}{2}} \frac{T^{2}}{M_{\mathrm{Pl}}}
$$

with the Planck mass $M_{\mathrm{Pl}} \simeq 10^{19} \mathrm{GeV}$ and the relativistic degrees of freedom $g_{*} \simeq 100$. From the equation,

$$
\Gamma \lesssim H(T)
$$



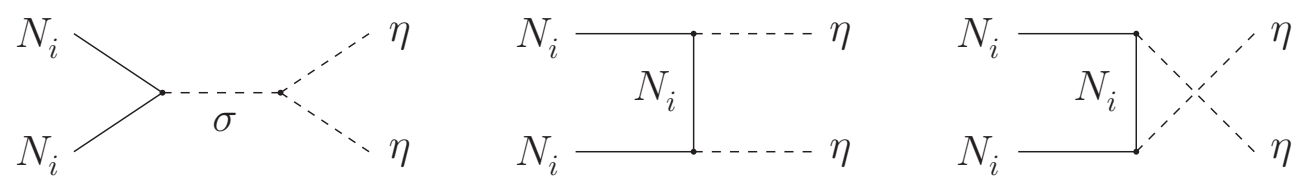

FIG. 1: The pair annihilation of the Majorana neutrinos into the Majorons.

we can find a solution to $T_{F}$ depending on $M_{N_{i}}$ and $f$.

For the purpose of analytical approximation, we expand the annihilation cross section $\sigma_{A}|\vec{v}|$ in powers of $\frac{1}{2}|\vec{v}| \leq 1$ for calculating its thermal average. Up to order $\mathcal{O}\left(|\vec{v}|^{2}\right)$, we arrive at

$$
\sigma_{A}|\vec{v}| \simeq \frac{1}{8 \pi}\left(\frac{1}{3}+\frac{\lambda_{3}^{2}}{4 \lambda_{1}^{2}}+\frac{5}{3 \lambda_{1}} \frac{M_{N_{i}}^{2}}{f^{2}}\right) \frac{M_{N_{i}}^{2}}{f^{4}}|\vec{v}|^{2}
$$

where the simplification, $s \ll M_{\sigma}^{2}$, has been adopted since the larger $s$ has no significant contribution to the thermal averaging in presence of the modified Bessel function $K_{1}\left(\frac{\sqrt{s}}{T}\right)$. For $\lambda_{1} \simeq \lambda_{3}=\mathcal{O}(1)$, the cross section (19) can be simplified as

$$
\sigma_{A}|\vec{v}| \simeq \frac{1}{8 \pi}\left(\frac{7}{12}+\frac{5}{3} \frac{M_{N_{i}}^{2}}{f^{2}}\right) \frac{M_{N_{i}}^{2}}{f^{4}}|\vec{v}|^{2} .
$$

Since the leptogenesis usually occurs at $M_{N_{i}} \gtrsim T_{l e p}>$ $T_{s p h} \sim 100 \mathrm{GeV}$, we only need satisfy the out-ofequilibrium condition (18) at $T=M_{N_{i}}$, i.e.

$$
\left.\Gamma \lesssim H(T)\right|_{T=M_{N_{i}}}
$$

Therefore we perform the equilibrium number density

$$
\left.n_{N_{i}}^{\mathrm{eq}}\right|_{T=M_{N_{i}}} \simeq 0.16 M_{N_{i}}^{3}
$$

and the thermal averaging

$$
\left.\left\langle\sigma_{A}|\vec{v}|\right\rangle\right|_{T=M_{N_{i}}} \simeq 0.023 \frac{M_{N_{i}}^{2}}{f^{4}} \text { with }\left.\left\langle|\vec{v}|^{2}\right\rangle\right|_{T=M_{N_{i}}} \simeq 1(23)
$$

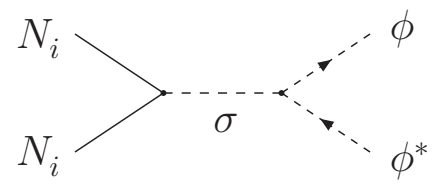

FIG. 2: The pair annihilation of the Majorana neutrinos into the pair of the SM Higgs.

Inputting (22) and (23) to (15) and then to (21) with (17), we eventually obtain the low bound on the breaking scale of the lepton number,

$$
\begin{aligned}
f & \gtrsim 0.1 \times\left(M_{N_{i}}^{3} M_{\mathrm{Pl}}\right)^{\frac{1}{4}} \\
& \gtrsim 0.1 \times\left(T_{s p h}^{3} M_{\mathrm{Pl}}\right)^{\frac{1}{4}} \\
& \simeq 10^{5} \mathrm{GeV} .
\end{aligned}
$$

In general, if we don't resort to the resonant effect 12, 13, 14 by highly fine tuning, $N_{i}$ should be heavier than $10^{9} \mathrm{GeV}$ [15, 16] to induce a desired CP asymmetry so that the lepton number should be broken at a much higher scale,

$$
f \gtrsim 10^{10} \mathrm{GeV}
$$

Our conclusion may be applied to the singlet-triplet Majoron model, where some triplet Higgs scalars $\xi$ with $L=-2$ are introduced to the SM as well as the previously singlet Higgs scalar $\chi$. Besides the quadratic and quartic interactions of these scalars, there will be additional lepton number conserving Yukawa and quartic 
interactions,

$$
\begin{aligned}
\mathcal{L} \supset & -\frac{1}{2} f_{i j} \overline{\psi_{L_{i}}^{c}} i \tau_{2} \xi \psi_{L_{j}}-\kappa \chi^{\dagger} \phi^{T} i \tau_{2} \xi \phi \\
& -\lambda\left(\chi^{\dagger} \chi\right) \operatorname{Tr}\left(\xi^{\dagger} \xi\right) .
\end{aligned}
$$

The last term of the above Lagrangian shows that the singlet's VEV will dominate the triplets' masses for certain range of the parameters. In this case, similar with the pair annihilations of the right-handed neutrinos in the singlet Majoron model, the scattering processes of the triplets to the Majoron and to the SM Higgs will also give us a low bound on the breaking scale of the lepton number. Obviously, this constraint is not as strong as that in the singlet Majoron model since the triplets' masses may be dominated by their mass term.

Let us now consider a R-parity violating supersymmetric model of thermal leptogenesis with right-handed neutrinos [17]. The essential terms in the superpotential are

$$
\begin{aligned}
\mathcal{W}= & \epsilon_{\alpha \beta}\left(Y_{\nu}\right)_{i j} \hat{N}_{i} \hat{L}_{j}^{\alpha} \hat{H}_{u}^{\beta}+\frac{1}{2} M_{N_{i j}} \hat{N}_{i} \hat{N}_{j} \\
& +\epsilon_{\alpha \beta} \lambda_{i} \hat{N}_{i} \hat{H}_{d}^{\alpha} \hat{H}_{u}^{\beta} .
\end{aligned}
$$

In the last term, lepton number is explicitly broken along with R-parity violation. The VEVs of the Higgs doublets would also induce a VEV to the scalar component of the right-handed neutrinos, which is of the order of $\left(Y_{\nu}\right)_{i j}\left\langle H_{u}\right\rangle\left\langle H_{d}\right\rangle / M_{N}$ and breaks the lepton number spontaneously. As a result, there will be a would-be Majoron in the model, which is very heavy. Unlike the previous discussions, these would-be Majorons could have lepton number violating interactions, that can constrain the scale of lepton number violation in this model. Consider the term in the scalar potential $\left|\frac{\partial \mathcal{W}}{\partial H_{u}}\right|^{2}$, which gives rise to the scattering of the Majorons $\tilde{N}+\tilde{N} \rightarrow \tilde{\nu} H$. This interaction will severely constrain the scale of leptogenesis in this class of models.

In summary, we have studied the constraint on the Majoron models if the leptogenesis is expected to explain the matter-antimatter asymmetry of the Universe. Our calculations indicate that the spontaneous breakdown of the global lepton number conservation in the singlet Majoron model should happen at a high scale $\left(\gtrsim 10^{5} \mathrm{GeV}\right)$ in order to guarantee the right-handed neutrinos to be out of equilibrium before the sphaleron action stops. This implies that some interesting phenomenology [18] of the singlet Majoron model will be absent from the LHC. We also point out that the similar constraint may exist in the singlet-triplet Majoron model and the R-parity violating supersymmetric Majoron model.

Acknowledgement: We would like to thank Professor Ernest Ma for his valuable comments.
[1] S. Weinberg, Phys. Rev. Lett. 43, 1566 (1979); E. Ma, Phys. Rev. Lett. 81, 1171 (1998).

[2] P. Minkowski, Phys. Lett. 67B, 421 (1977); T. Yanagida, in Proc. of the Workshop on Unified Theory and the Baryon Number of the Universe, ed. O. Sawada and A. Sugamoto (KEK, Tsukuba, 1979), p. 95; M. Gell-Mann, P. Ramond, and R. Slansky, in Supergravity, ed. F. van Nieuwenhuizen and D. Freedman (North Holland, Amsterdam, 1979), p. 315; S.L. Glashow, in Quarks and Leptons, ed. M. Lévy et al. (Plenum, New York, 1980), p. 707; R.N. Mohapatra and G. Senjanović, Phys. Rev. Lett. 44, 912 (1980).

[3] M. Magg and C. Wetterich, Phys. Lett. B 94, 61 (1980); J. Schechter and J.W.F. Valle, Phys. Rev. D 22, 2227 (1980); T.P. Cheng and L.F. Li, Phys. Rev. D 22, 2860 (1980); G. Lazarides, Q. Shafi, and C. Wetterich, Nucl. Phys. B 181, 287 (1981); R.N. Mohapatra and G. Senjanović, Phys. Rev. D 23, 165 (1981).

[4] M. Fukugita and T. Yanagida, Phys. Lett. B 174, 45 (1986).

[5] R.N. Mohapatra and X. Zhang, Phys. Rev. D 46, 5331 (1992).

[6] E. Ma and U. Sarkar, Phys. Rev. Lett. 80, 5716 (1998).
[7] T. Hamby and G. Senjanović, Phys. Lett. B 582, 73 (2004).

[8] V.A. Kuzmin, V.A. Rubakov, and M.E. Shaposhnikov, Phys. Lett. B 155, 36 (1985).

[9] Y. Chikashige, R.N. Mohapatra, and R.D. Peccei, Phys. Lett. B 98, 265 (1981).

[10] E.W. Kolb and S. Wolfram, Nucl. Phys. B 172, 224 (1980).

[11] M.A. Luty, Phys. Rev. D 45, 455 (1992).

[12] M. Flanz, E.A. Paschos, and U. Sarkar, Phys. Lett. B 345, 248 (1995).

[13] M. Flanz, E.A. Paschos, U. Sarkar, and J. Weiss, Phys. Lett. B 389, 693 (1996).

[14] A. Pilaftsis, Phys. Rev. D 56, 5431 (1997).

[15] S. Davidson and A. Ibarra, Phys. Lett. B 535, 25 (2002).

[16] W. Buchmüller, P. Di Bari, and M. Plümacher, Nucl. Phys. B 665, 445 (2003).

[17] Y. Farzan and J.W.F. Valle, Phys. Rev. Lett. 96, 011601 (2006).

[18] For the implications of singlet Majoron, see: G. Jungman and M.A. Luty, Neucl. Phys. B 361, 24 (1991); and references therein. 